Z. Klin. Chem. Klin. Biochem.

13. Jg. 1975 , S. $341-349$

\title{
Verbesserte Trihydroxyindolmethode zur Bestimmung der Harnkatecholamine
}

\author{
Von $U$. Werner
}

Abteilung für Nieren- und Hochdruckkranke der Medizinischen Klinik und Poliklinik des Universitätsklinikum Essen

(Eingegangen am 22. November 1974/11. März 1975)

\section{Hern Prof. Dr. O. H. Arnold zum 65. Geburtstag gewidmet}

Zusammenfassung: Zur Katecholaminabtrennung im Harn wurden kommerzielle Ionenaustauschchromatographiesäulen verwandt. Die Katecholaminbestimmung erfolgte fluorimetrisch mit einer neuen Trihydroxyindolmethode. Durch kombinierte Anwendung von Borsäure, Kupferionen, Mercaptoäthanol und finaler Ansäuerung konnte die Fluoreszenzausbeute im Vergleich zu anderen Trihydroxyindolmethoden für Adrenalin und Noradrenalin gesteigert werden. Die Fluorophore sind sehr stabil: Der Fluoreszenzverlust von Adrenolutin betrug während 180 Minuten 22\%, bei Noradrenolutin war kein Verlust nachweisbar. Zur Differenzierung der Amine wird Adrenalin bei pH 2,85 und Noradrenalin bei pH 7 oxidiert. Die Kriterien für eine Analysenmethode werden in bezug auf Empfindlichkeit, E.ichtigkeit, Präzision und Spezifität erfüllt.

Als Normwerte wurden an einem Kollektiv von 17 gesunden Probanden für Adrenalin ( $\bar{x} \pm \mathrm{s}) 44,8 \pm 16,9 \mathrm{nmol} /$ $24 \mathrm{~h}(8,2 \pm 3,1 \mu \mathrm{g} / 24 \mathrm{~h})$ und für Noradrenalin $(\overline{\mathrm{x}} \pm \mathrm{s}) 224,0 \pm 68,0 \mathrm{nmol} / 24 \mathrm{~h}(37,9 \pm 11,5 \mu \mathrm{g} / 24 \mathrm{~h})$ gefunden.

Die gleichzeitige Verabfolgung von $\alpha$-Methyldopa $(2 \mathrm{~g} / \mathrm{d})$ bei 13 Patienten mit primärer Hypertonie störte die fluorimetrische Bestimmung der Katecholamine nicht.

\section{An improved trihydroxyindole method for the determination of urinary catecholamines}

Summary: Commercially available columns for ion exchange chromatography were used for the separation of catecholamines in urine. The estimation of catecholamines was performed fluorimetrically by a new trihydroxyindole method. The fluorescence of adrenaline and noradrenaline was enhanced, in comparison with other methods, by the combined application of boric acid, copper-ions, mercaptoethanol and final reacidification.

The fluorophores are stable: The loss of fluorescence of adrenolutin amounted to $22 \%$ during 180 minutes, and there was no loss of noradrenolutin. For the differentiation of amines, adrenaline was oxidized at $\mathrm{pH} 2.85$ and noradrenaline at $\mathrm{pH}$ 7. Precision, accuracy, sensitivity and specifity fulfilled the criteria of analysis.

The normal values, determined in a collective of 17 healthy persons, were adrenaline $(\bar{x} \pm s) 44.8 \pm 16.9 \mathrm{nmol} / 24 \mathrm{~h}$ and noradrenaline $(\overline{\mathrm{x}} \pm \mathrm{s}) 224.0 \pm 68.0 \mathrm{nmol} / 24 \mathrm{~h}$. The simultaneous application of $\alpha$-methyldopa $(2 \mathrm{~g} / \mathrm{day})$ in 13 patients with primary hypertension did not distrub the fluorimetric estimation of catecholamines.

\section{Einführung}

Über die fluorimetrische Bestimmung von Noradrenalin und Adrenalin mit der Trihydroxyindolmethode sind zahlreiche Modifikationen publiziert worden (Übersicht bei Udenfriend(1)). Das Prinzip der Methode besteht darin, daß Adrenalin bzw. Noradrenalin zu Adrenochrom bzw. Noradrenochrom oxidiert werden und diese in stark alkalischem Milieu in die entsprechenden Fluorophore Adrenolutin und Noradrenolutin übergeführt werden.

Diese Lutine sind im alkalischen Milieu nicht stabil.

Um einen Zerfall innerhalb von Minuten $(2,3)$ zu verhindern, werden Reduktionsmittel zugefügt. Als gutes Reduktionsmittel hat sich Ascorbinsäure bewährt.
Ascorbinsäure hat jedoch den Nachteil, selbst in fluoreszierende Produkte zu zerfallen. Daher wurden verschiedene Stoffe zum Schutz der Ascorbinsäure beigefügt, z. B. Ä thylendiamin (4) oder $\beta$-Thiopropionsäure (5).

Häggendal (6) ersetzte die Ascorbinsäure durch 2,3Dimercaptopropanol (BAL) und erhielt eine hohe stabile Fluoreszenz bei niedrigen Leerwerten. WeilMalherbe \& Bigelow (7) benutzten Dimercaptoäthanol und konnten durch Ansäuern auf pH 5 eine weitere Steigerung der Stabilität und Empfindlichkeit erreichen. Dabei ist für die Adrenalinbestimmung der Zusatz von Kupferionen erforderlich, die beim Ansäuern ein Präzipitat bilden, das abzentrifugiert werden $\operatorname{muß}(7,8)$. 
Nachstehend wird eine Modifikation der Trihydroxyindolmethode beschrieben, die mit dem Ziel entwickelt wurde, einerseits praktikabel und zeitsparend zu sein, andererseits eine hohe spezifische Fluoreszenzausbeute zu gewährleisten.

\section{Material und Methoden \\ Untersuchungsgut}

Die 24 Stunden-Sammelurine von 17 normotonen Kontrollpersonen, 13 Hypertonikern, die $9,5 \mathrm{mmol}(2 \mathrm{~g}) \alpha$-Methyldopa täglich per os erhielten und einem Patienten mit einem Phäochromocy tom wurden untersucht.

\section{Reagenzien}

1. Borsäure, 0,67 mol/l (Merck Art. 165)

2. Natriumhydroxid, $0,2 \mathrm{~mol} / \mathrm{l}$ (Merck Art. 6498)

3. EDTA $26,86 \mathrm{mmol} / \mathrm{l}(10 \mathrm{~g} / \mathrm{l})$ (Merck Art. 8418)

4. Ameisensäure, 2,0 mol/l (Merck Art. 264)

5. Kaliumhexacyanoferrat[III] $7,59 \mathrm{mmol} / \mathrm{l}(2,5 \mathrm{~g} / \mathrm{l})$ (Merck Art. 4973)

6. Kupferacetat, $1 \mathrm{mmol} / \mathrm{l}$ (Merck Art. 2711)

7. Reduktionsreagenz:

a) Mercaptoäthanol, $0,709 \mathrm{~mol} / 1$ ( $50 \mathrm{ml} / \mathrm{l})$ (Serva Art. 28625)

b) Natriumsulfit, $1,587 \mathrm{~mol} / \mathrm{l}(200 \mathrm{~g} / \mathrm{l})$ (Merck Art. 6657)

c) Natriumhydroxid, $10 \mathrm{~mol} / 1$ (Merck Art. 6498)

Gleiche Volumina von $\mathrm{a}, \mathrm{b}$ und $\mathrm{c}$ wurden gemischt.

Das Reduktionsreagenz ist im geschlossenen Gefäß bei

Raumtemperatur für 2 Wochen stabil.

8. Essigsäure, $10 \mathrm{~mol} / 1$ (Merck Art. 90063)

9. Boratpuffer, $0,67 \mathrm{~mol} / \mathrm{l}, \mathrm{pH} 2-10$

Borsäure (Reagenz Nr. 1) wurde mit Ameisensäure bzw.

Natriumhydroxidlösung elektrometrisch auf den entsprechenden $\mathrm{pH}$-Wert eingestellt.

10. Adrenalin und Noradrenalin-Stammlösung:

$54,6 \mu \mathrm{mol}(10 \mathrm{mg})$ Adrenalin bzw. $59,1 \mu \mathrm{mol}(10 \mathrm{mg})$ Noradrenalin wurden in $1 \mathrm{ml}$ Salzsäure, $1 \mathrm{~mol} / 1$, gelöst und mit deionisiertem Wasser auf $100 \mathrm{ml}$ aufgefüllt und kühl aufbewahrt. Arbeitsstandard: Stammlösung 1: 100 verdünnen, täglich frisch ansetzen, im Eisbad halten.

Feinsubstanzen

Noradrenalin

Adrenalin

Normetanephrin- $\mathrm{HCl}$

Metanephrin- $\mathrm{HCl}$

3,4 Dihydroxynorephedrin- $\mathrm{HCl}$

3,4 Dihydroxyphenylglycol

3-Methoxy-4-hydroxyphenyläthylamin-

hydrochlorid

$\alpha$-Methyl-3,4-dihydroxy-phenylalanin

3,4-Dihydroxyphenylalanin

Serotonin-Hydrogenmaleinat

Tyramin- $\mathrm{HCl}$

$L$-Histidin- $\mathrm{HCl}$

$D, L$-Tyrosin

3,4-Dihydroxyphenylessigsäure

3-Methoxy-4-hy droxyphenyläthylen-

glycol-Piperazinsalz

Histaminiumdihydrochlorid

Vanillinsäure

Vanillinmandelsäure

Homovanillinsäure

$D, L$-Octopamin- $\mathrm{HCl}$

$D, L-3,4-D$ ihydroxymandelsäure

Dopamin-.iydrochlorid
(Hoechst) ${ }^{1}$ )

(Hoechst) $^{1}$ )

(Hoechst) ${ }^{1}$ )

(Hoechst) ${ }^{1}$ )

(Calbiochem 3063)

(Calbiochem 308144)

(Calbiochem 45426)

(Hoechst) ${ }^{1}$ )

(Merck 4196)

(Fluka 85040)

(Merck 8373)

(Serva 24830)

(Serva 37520)

(Fluka 37860)

(Calbiochem 454205)

(Merck 4370)

(Roth 4884)

(Calbiochem 4542)

(Calbiochem 38588)

(Calbiochem 49444)

(Calbiochem 3062)

(Fluka 56610)

\section{Geräte und Ausrüstung}

Kationenaustauschharz-Chromatographiesäulen $(0,7 \times 5,0 \mathrm{~cm})$ der Firma Bio-Rad Laboratories, München; Amberlite CG 50,

1) Wir danken Frau Dr. Busch, Arzneimittelkontor der Firma Hoechst, Köln, für die freundliche Überlassung von Versuchsmengen.
$\left(\mathrm{H}^{+}\right), 200-400$ mesh der Firma Serva, Heidelberg; Polystryrolröhrchen (16/100, glasklar) der Firma Greiner, Nürtingen; Eppendorf-Pipetten der Firma Eppendorf.

Die fluorimetrische Bestimmung erfolgte mit einem SPF Aminco Bowman Fluorometer mit einem „Condensing mirror system" (American Instrument Company, Silver Springs, Md. USA). Schlitzweite $2 \mathrm{~mm}$. Xenon Lampe: Osram XBO $150 \mathrm{~W} / 1$. Rechteckquarzküvetten $1 \times 1 \times 4,5 \mathrm{~cm}$. Die Empfindlichkeit wurde täglich justiert mit einer Chininsulfatlösung ( $1 \mathrm{mg} / 1$ in $0,1 \mathrm{~mol} / 1$ Schwefelsäure) bei einer Wellenlänge von $345 / 450 \mathrm{~mm}$ (Anregung/Emission) und einer Standardbeśtimmung von 59 pmol Noradrenalin nach der Methode von Häggendal (6).

\section{Arbeitsvorschrift}

\section{Säulenchromatographie}

Der 24-Stunden-Urin wird in einem Gefäß mit $15 \mathrm{ml}$ konzentrierter Salzsäure gesammelt. Etwa $30 \mathrm{ml}$ Urin werden scharf abzentrifugiert und $5 \mathrm{ml}$ davon für die Katecholaminbestimmung entnommen. Der Rest wird für Doppelbestimmungen und für die Bestimmung der Metabolite kühl aufbewahrt.

\section{Ausführung}

Der Urin $(5 \mathrm{ml})$ wird mit Natronlauge $(0,2 \mathrm{~mol} / \mathrm{l})$ auf $\mathrm{pH} 6,5$ eingestellt und mit EDTA (14 ml, 26,86 mmol/l) versetzt. Der so vorbereitete Harn wird über die Säule gegeben. Die Säule wird mit deionisiertem Wasser $(3 \times 7,5 \mathrm{ml})$ nachgespült. Die Katecholamine werden mit Borsäure $(10 \mathrm{ml}, 0,67 \mathrm{~mol} / \mathrm{l})$ eluiert.

Die fluorimetrische Bestimmung wird am gleichen Tag vorgenommen.

\section{Bestimmung und Berechnung}

Die Reihenfolge für die Präparation der Probe ist zu ersehen aus Tabelle 1. Für die Berechnung der Katecholamine und ihrer Differenzierung in Adrenalin und Noradrenalin gibt es mehrere Möglichkeiten, von denen hier zwei ausführlicher dargistellt werden.

1. Eine exakte Berechnung ist möglich, wenn die Methoden von Crout (9) oder Weil-Malherbe \& Bigelow (7) auf die veränderten Volumina entsprechend angewandt werden (Tab. 1). Sie setzt voraus, daß bei $\mathrm{pH} \mathrm{2,85}$ nur Adrenalin oxidiert wird. Sollte mehr als $5 \%$ der Gesamtfluoreszenz auf Noradrenalin zurückgehen, ist eine der optischen Differenzierungsmethoden anzuwenden $(7,16)$.

2. Eine vereinfachte Form der Berechnung ist möglich, wenn Eichgeraden für Adrenalin und Noradrenalin erstellt werden. Es wurden zu je $5 \mathrm{ml}$ Poolurin 0;2,73; $8,19 \mathrm{nmol}$ Adrenalin sowie $0 ; 2,95 ; 8,87 \mathrm{nmol}$ Noradrenalin zugegeben. Von den erhaltenen Fluoreszenzeinheiten werden die Fluoreszenzeinheiten der O-Werte substrahiert und die Differenz auf Millimeterpapier aufgetragen. Voraussetzung für ein solches Vorgehen ist die tägliche Eichung des Fluorometers.

Adrenalin (nmol/l): Anhand der Nettofluoreszenzwerte $\left(\mathrm{S}_{3}-\mathrm{FB}_{3}\right)$ der Harnprobe kann die Adrenalinkonzentration des Harns direkt von der Eichgeraden abgelesen werden.

Noradrenalin (nmol/1): Das Vorgehen bei Noradrenalin ist ähnlich wie bei Adrenalin. Das gefundene Adrenalin wird mit dem Faktor

$$
\frac{A_{7}(390 / 470)}{A_{3}(415 / 490)}
$$

multipliziert, das Produkt von der Gesamtfluoreszenz der bei pH 7 oxidierten Probe subtrahiert. Der Faktor liegt bei 0,7.

\section{Ergebnisse}

\section{Linearität}

Wenn Adrenalin bei $\mathrm{pH} 2,85$ und Noradrenalin bei $\mathrm{pH} 7$ oxidiert wurden, konnte ein Linearitätsbereich der Fluoreszenzintensität in einer Konzentration von $1 \mathrm{nmol}$ bis 1 pmol pro Probe nachgewiesen werden. 
Tab. 1. Probenansatz

\begin{tabular}{|c|c|c|c|c|c|c|c|c|}
\hline & $S_{3}$ & $\mathbf{F B}_{\mathbf{3}}$ & $\mathrm{SA}_{3}$ & $\mathrm{SN}_{3}$ & $\mathrm{~S}_{7}$ & $\mathrm{FB}_{7}$ & $\mathrm{SA}_{7}$ & $\mathrm{SN}_{7}$ \\
\hline Boratpuffer, $0,67 \mathrm{~mol} / 1, \mathrm{pH} 7(\mathrm{ml})$ & - & - & - & - & 0,10 & 0,10 & 0,10 & 0,10 \\
\hline Borsaures Eluat (ml) & 0,70 & 0,70 & 0,70 & 0,70 & 0,70 & 0,70 & 0,70 & 0,70 \\
\hline Standard (a) (ml) & - & - & 0,01 & 0,01 & - & - & 0,01 & 0,01 \\
\hline Ameisensäure $2,00 \mathrm{~mol} / \mathrm{l}(\mathrm{ml})$ & 0,10 & 0,10 & 0,10 & 0,10 & - & - & - & - \\
\hline Kupferacetat $1 \mathrm{mmol} / \mathrm{l}(\mathrm{ml})$ & 0,05 & 0,05 & 0,05 & 0,05 & 0,05 & 0,05 & 0,05 & 0,05 \\
\hline $\mathrm{K}_{3}\left[\mathrm{Fe}(\mathrm{CN})_{6}\right] 7,59 \mathrm{mmol} / 1(\mathrm{ml})$ & 0,05 & 0,05 & 0,05 & 0,05 & 0,05 & 0,05 & 0,05 & 0,05 \\
\hline Einwirkzeit (min) & 3 & 3 & 3 & 3 & 3 & 3 & 3 & 3 \\
\hline Natriumhydroxid $10 \mathrm{~mol} / \mathrm{l}(\mathrm{ml})$ & - & 0,10 & - & - & - & 0,10 & - & - \\
\hline Reduktionsreagenz & 0,20 & - & 0,20 & 0,20 & 0,20 & - & 0,20 & 0,20 \\
\hline Einwirkzeit (min) & 3 & 10 & 3 & 3 & 3 & 10 & 3 & 3 \\
\hline Essigsäure $10 \mathrm{~mol} / 1(\mathrm{ml})$ & 0,20 & 0,20 & 0,20 & 0,20 & 0,20 & 0,20 & 0,20 & 0,20 \\
\hline Reduktionsreagenz (ml) & - & 0,10 & - & - & - & 0,10 & - & $-\infty$ \\
\hline Volumen (ml) & 1,30 & 1,30 & 1,31 & 1,31 & 1,30 & 1,30 & 1,31 & 1,31 \\
\hline
\end{tabular}

Die Lösungen werden in der Reihenfolge von oben nach unten zusammengegeben.

a) Standard enthält $5,46 \mu \mathrm{mol} / 1(1 \mathrm{mg} / \mathrm{l})$ Adrenalin (für $\mathrm{SA}_{3}$ und $\mathrm{SA}_{7}$ ) bzw. $5,91 \mu \mathrm{mol} / 1(1 \mathrm{mg} / 1)$ Noradrenalin (für $\mathrm{SN}_{3}$ und $\mathrm{SN}_{7}$ )

$\mathrm{S}_{3}=$ Probe (Sample) pH 2,85

$\mathrm{FB}_{3}=$ Leerwert (faded blank) pH 2,85

$\mathrm{SA}_{3}=$ Innerer (added) Standard Adrenalin pH 2,85

$\mathrm{SN}_{3}=$ Innerer (added) Standard Noradrenalin $\mathrm{pH} 2,85$

$\mathrm{S}_{7}=$ Probe (Sample) $\mathrm{pH} 7$

$\mathrm{FB}_{7}=$ Leerwert (faded blank) $\mathrm{pH} 7$

$\mathrm{SA}_{7}=$ Innerer (added) Standard Adrenalin $\mathrm{pH} 7$

$\mathrm{SN}_{7}=$ Innerer (added) Standard Noradrenalin pH 7

$A_{S}=$ pmol Adrenalin pro Probe (Sample)

$\mathrm{N}_{\mathrm{S}}=$ pmol Noradrenalin pro Probe (Sample)

$\mathrm{A}_{3}=$ Nettofluoreszenz von $55 \mathrm{pmol}(10 \mathrm{ng})$,, added " Standard Adrenalin pH 2,85 $=\mathrm{SA}_{3}-\mathrm{S}_{3}$

$\mathrm{A}_{7}=$ Nettofluoreszenz von $55 \mathrm{pmol}(10 \mathrm{ng})$,,added" Standard Adrenalin $\mathrm{pH} 7=\mathrm{SA}_{7}-\mathrm{S}_{7}$

$\mathrm{N}_{7}=$ Nettofluoreszenz von $59 \mathrm{pmol}(10 \mathrm{ng})$,,added" Standard Noradrenalin $\mathrm{pH} 7=\mathrm{SN}_{7}-\mathrm{S}_{7}$

$A_{S}=\frac{54,6 \cdot\left(S_{3}-F_{3}\right)}{A_{3}}$

$\mathrm{N}_{\mathrm{S}}=\frac{59,1 \cdot\left(\mathrm{S}_{7}-\mathrm{FB}_{7}\right)-\mathrm{A}_{\mathrm{S}} \cdot \mathrm{A}_{7}}{\mathrm{~N}_{7}}$

\section{Stabilität}

Der gleichzeitige Gebrauch von Kupferionen und Boratpuffer führte zu einer sehr guten Stabilität von Noradrenalin und einer guten Stabilität von Adrenalin (Abb. 1). Der Abfall der Fluoreszenz von Adrenalin war nach 60 min $10 \%$, nach 120 min $16 \%$ und nach 180 min $22 \%$. Wurde Noradrenalin bei pH 7 ohne $\mathrm{Cu}^{+}$ oxidiert, war ein ,fading" nachweisbar, ähnlich wie bei Adrenalin. Keine Veränderung der Fluoreszenz konnte bei Noradrenalin und den "faded blanks" von beiden Katecholaminen nachgewiesen werden.

\section{Einfluß des $\mathrm{pH}$-Wertes}

Es wurde der Einfluß des pH (Boratpuffer $0,67 \mathrm{~mol} / \mathrm{l}$ ) während der Oxidation auf die Bildung der Fluorophore geprüft. Während Adrenalin bei $\mathrm{pH} 3$ schon eine volle Fluoreszenzintensität entwickelte, war Noradrenalin noch nicht oxidiert. Noradrenalin hatte ein Fluoreszenzmaximum bei $\mathrm{pH} 5$. Zwischen $\mathrm{pH} 6$ und $\mathrm{pH} 10$ waren nur geringe Unterschiede zwischen beiden Aminen zu beobachten. Wurde Adrenalin bei einer Wellenlänge von 390/470 nm (Anregung/Emission) abgelesen, so wurden noch etwa $2 / 3$ der Fluoreszenzausbeute gefunden.

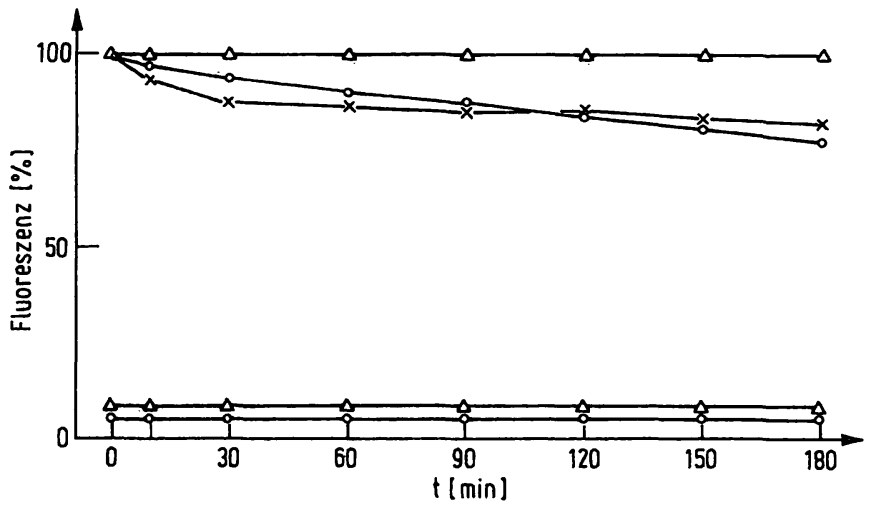

Abb. 1. Stabilität der Fluoreszenzprodukte. 55 pmol Adrenalin bzw. 59 pmol Noradrenalin wurden pro Ansatz in Boratpuffer $0,67 \mathrm{~mol} / \mathrm{l}$ beigegeben. Adrenalin ( 0 ) AdrenalinLeerwert $(0)$ wurden bei $\mathrm{pH} 2,85$ oxidiert, und Noradrenalin-Leerwert $(\Delta)$ wurden bei $\mathrm{pH} 7$ oxidiert.

Noradrenalin ohne $\mathrm{Cu}^{++}(\mathrm{x})$, Oxidation bei $\mathrm{pH}$ 7. Fluoreszenz wurde sofort nach der Essigsäurezugabe gemessen und auf $100 \%$ gesetzt. 


\section{Stabilität der Katecholamine im Boratpuffer}

Es wurden Standards von $5,46 \mu \mathrm{mol} / 1$ Adrenalin bzw. $5,91 \mu \mathrm{mol} / 1$ Noradrenalin in Boratpuffer $0,67 \mathrm{~mol} / 1$ und Acetatpuffer $0,67 \mathrm{~mol} / 1, \mathrm{pH} \mathrm{3,6,7,8}$ und 9 hergestellt (Tab. 2). Daraufhin wurden Proben von jedem Standard entnommen und die Fluoreszenz gemessen.

Tab. 2. Stabilität von Adrenalin und Noradrenalin Boratpuffer $0,67 \mathrm{~mol} / \mathrm{l}$, Acetatpuffer $0,67 \mathrm{~mol} / 1$. Konzentrationen von Adrenalin $5,46 \mu \mathrm{mol} / 1 \mathrm{bzw}$. Noradrenalin $5,91 \mu \mathrm{mol} / \mathrm{l}$ entsprechen $100 \%$. Wiederfindung nach Lagerung von 7 Tagen bei Raumtemperatur $\left(25^{\circ} \mathrm{C}\right)$.

\begin{tabular}{lcccc}
\hline & Boratpuffer & \multicolumn{3}{c}{ Acetatpuffer } \\
Adrenalin & $\begin{array}{l}\text { Nor- } \\
\text { adrenalin }\end{array}$ & Adrenalin & $\begin{array}{l}\text { Nor- } \\
\text { adrenalin }\end{array}$ \\
\hline pH 3 & $100 \%$ & $100 \%$ & $74 \%$ & $69 \%$ \\
pH 6 & $92 \%$ & $92 \%$ & $14 \%$ & $48 \%$ \\
pH 7 & $88 \%$ & $85 \%$ & $0 \%$ & $14 \%$ \\
pH 8 & $88 \%$ & $81 \%$ & $0 \%$ & $0 \%$ \\
pH 9 & $75 \%$ & $70 \%$ & $0 \%$ & $0 \%$ \\
\hline
\end{tabular}

Die Standards blieben 7 Tage bei Raumtemperatur von $25^{\circ} \mathrm{C}$ stehen. Die nun durchgeführte Kontrollbestimmung eines jeden Standards ergab eindeutige Unterschiede zwischen Boratpuffer und Acetatpuffer. Boratpuffer schützt vor oxidativem Abbau. Der Verlust der Katecholamine war außerdem abhängig vom $\mathrm{pH}$-Wert und am geringsten im sauren Bereich.

In Boratpuffer ist der Katecholaminverlust auch im alkalischen Bereich gering, in Acetatpuffer jedoch vollständig.

In Urineluaten (pH 7) ist der Abfall des Adrenalinanteils rascher als in Boratpuffer, er variiert jedoch von Eluat zu Eluat. Es ist deshalb empfehlenswert, die Bestimmung sofort vorzunehmen oder die Eluate kühl zu lagern, um die Verluste gering zu halten.

Der Einfluß von Kupferionen und finaler Ansäuerung

ist in Tabelle 3 zu sehen. Der Effekt von Kupferionen ist besonders deutlich beim Adrenalin. Kupfer ist hier unbedingt erforderlich, gleich, ob die Oxidation bei pH 2,85 oder pH 7 durchgeführt wird. Die Steigerung der Fluoreszenz ist bis zum 25-fachen des Ausgangswertes (A7: Adrenalin pH 7). Die finale Ansäuerung führt zu einer nochmaligen Steigerung der Fluoreszenz (2-fach bei A7, 3-fach bei A3).

Noradrenalin zeigt, bei pH 7 oxidiert, auch ohne Kupfer mit Ansäuern eine hohe Fluoreszenz. Mit Kupfer ist hier ebenfalls eine deutliche Steigerung (etwa 1,7-fach) der Fluoreszenz zu zeigen. Bis zu einer Konzentration von $1 \mathrm{mmol} / 1 \mathrm{Kupferacetat,} 50 \mu \mathrm{l}$ je Probe, war eine̊ Zunahme der Fluoreszenzintensität nachweisbar.
Tab. 3. Einfluß von Kupferionen und finaler Ansäuerung auf die Fluoreszenzausbeute in Fluoreszenzeinheiten Jede Probe enthielt 55 pmol Adrenalin oder $59 \mathrm{pmol}$ Noradrenalin in Boratpuffer $0,67 \mathrm{~mol} / 1$.

$\mathrm{A}_{3}$ : Adrenalin oxidiert bei pH 2,85

$\mathrm{A}_{7}$ : Adrenalin oxidiert bei $\mathrm{pH} 7$

$\mathrm{N}_{7}$ : Noradrenalin oxidiert bei $\mathrm{pH} 7$

\begin{tabular}{lccc}
\hline & \multicolumn{3}{c}{ Fluoreszenzausbeute } \\
\hline Prozedur & A 3 & A 7 & N 7 \\
$\begin{array}{l}\text { ohne } \mathrm{Cu}^{++} \\
\text {ohne } \mathrm{Ansäuerung}\end{array}$ & 5 & 3 & 23 \\
$\begin{array}{l}\text { ohne } \mathrm{Cu}^{++} \\
\text {mit } \mathrm{Ansäuerung} \\
\text { mit } \mathrm{Cu}^{++}\end{array}$ & 13 & 10 & 98 \\
$\begin{array}{l}\text { ohne Ansäuerung } \\
\text { mit } \mathrm{Cu}+\end{array}$ & 59 & 77 & 92 \\
mit Ansäuerung & 180 & 165 & 178 \\
\hline
\end{tabular}

Höhere Konzentrationen führten zu höheren Leerwerten, und ein flockiges Präzipitat fiel aus.

Der pH-Wert nach Ansäuern liegt zwischen 4,5 und 4,8. Wurde statt einer Essigsäure $10 \mathrm{~mol} / 1$ eine Essigsäure $5 \mathrm{~mol} / \mathrm{l}$ verwandt, liegt der $\mathrm{pH}$-Wert bei 5,0. Dabei kam es zu keiner meßbaren Veränderung der Fluoreszenz, jedoch war die Stabilität der Fluoreszenzprodukte geringer. Deshalb wurde eine Essigsäure $10 \mathrm{~mol} / \mathrm{l}$ bevorzugt.

\section{Kaliumhexacyanoferrat[III] und Leerwert}

Die verwendete Konzentration von Ferricyanid ergab optimale Resultate. Reduzierung der Kaliumhexacyanoferrat (III)-Konzentration maximal um den Faktor 50 führte zu einer geringen Steigerung der Fluoreszenz von Noradrenalin bei einem geringen Rückgang der Leerwerte. Adrenalin, bei pH 2,85 oxidiert, zeigte einen deutlichen Rückgang der Fluoreszenz.

\section{Oxidationszeit und Temperatureinflüsse}

Es wurden die Fluoreszenzausbeuten bei Oxidationszeiten von 1, 3 und 5 min über einen Bereich von $\mathrm{pH}$ 2-10 geprüft. Eine Verminderung der Fluoreszenz wurde nur für Adrenalin bei $\mathrm{pH} 3$ und 1 min Oxidationszeit festgestellt, so daß 3 min Oxidationszeit als ausreichend angesehen wurden. Wurde die Oxidation im Eisbad durchgeführt, war die Bildung von Adrenolutin bei pH 3 stark herabgesetzt. Eine Verlängerung der Oxidationszeit auf $5 \mathrm{~min}$ brachte auch hier keine besseren Ergebnisse. Die Oxidation sollte bei Raumtemperatur durchgeführt werden. Wurden die Proben nach Beendigung der Prozedur kühl gehalten, so erhöhte sich die Fluoreszenz erheblich.

\section{Reduktionsgemisch}

Das Reduktionsgemisch enthält Mercaptoäthanol, Natronlauge und Natriumsulfit (s. methodischer Teil). 
Im vorliegenden Mischungsverhältnis waren optimale Fluoreszenzen zu erhalten, wenn Boratpuffer verwendet wurde. Bei Acetatpuffer gleicher Molarität war die Fluoreszenzausbeute etwa $1 / 3$ geringer, höhere Natriumhydroxidkonzentration konnte hier die Fluoreszenzausbeute gering verbessern. Mercaptoäthanol in einer Konzentration von $0,709 \mathrm{~mol} / \mathrm{l}$ ergab ein günstiges Verhältnis zwischen Fluoreszenz- und Leerwert. Diese relativ hohe Konzentration von Mercaptoäthanol sichert die lange Haltbarkeit des fertigen Reduktionsgemisches. Höhere Konzentrationen ergaben hohe Leerwerte. Die Gegenivart von Sulfit war für eine hohe Fluoreszenzausbeute unabdingbar.

\section{Andere Elutionsmedien}

Um den Einfluß des Elutionsmediums auf die Fluoreszenzausbeute zu prüfen, wurde neben borsaurem Leereluat essigsaures Leereluat erstellt und dann Adrenalin bzw. Noradrenalin zugegeben. Bei einem „Leereluat" wurde die Säulenprozedur ohne Harn durchgeführt. Wie aus Tabelle $4 \mathrm{zu}$ sehen ist, ist die Fluoreszenzausbeute bei borsaurem Eluat besonders günstig, gleich, ob der Ionenaustauscher der Fertigsäulen oder Amberlite CG 50 verwendet wurden. Bei essigsäurem Eluat erweist sich die niedrigere Molarität als günstiger.

Tab. 4. Fluoreszenzausbeute in Leereluaten

Jeder Probe wurden $55 \mathrm{pmol}$ (10 ng) Adrenalin bzw. 59 pmol (10 ng) Noradrenalin zugesetzt. Der OxidationspH wurde elektrometrisch eingestellt. Dic Fluoreszenzausbeute von Noradrenalin im borsauren Eluat der Fertigsäulen wurden auf 100 gesetzt.

\begin{tabular}{llcc}
\hline Säule & & $\mathrm{pH}$ & Fluoreszenz \\
\hline $\begin{array}{l}\text { Fertigsäule } \\
\text { eluiert mit }\end{array}$ & $\begin{array}{l}\text { Adrenalin } \\
\text { Borsäure 0,67 mol/1 }\end{array}$ & 3 & 86 \\
Noradrenalin & 7 & 100 \\
$\begin{array}{l}\text { Amberlite C G 50 } \\
\text { eluiert mit }\end{array}$ & Adrenalin & 3 & 83 \\
Borsäure 0,67 mol/1 & Noradrenalin & 7 & 100 \\
$\begin{array}{lll}\text { Amberlite C G 50 } \\
\text { eluiert mit }\end{array}$ & Adrenalin & 3 & 38 \\
$\begin{array}{l}\text { Essigsäure 1 mol/1 } \\
\text { Nluminiumoxid }\end{array}$ & Noradrenalin & 6 & 61 \\
eluiert mit & Adrenalin & 3 & 53 \\
Essigsäure 0,2 mol/1 & Noradrenaiin & 6 & 76 \\
\hline
\end{tabular}

Fluoreszenzausbeute im Vergleich zu anderen Trihydroxyindolmethoden

Zum Vergleich wurden eine alkalische Trihydroxyindolmethode (2), die auch vom Hersteller der Fertigsäulen empfohlen wird, eine reacidifizierende Thiol-Trihydroxyindolmethode (7) und die vorliegende Methode herangezogen. Es wurden zu jeder Methode die entsprechenden Leereluate erstellt und jeder Probenansatz mit 55 pmol Adrenalin oder 59 pmol Noradrenalin aufgestockt. Dieses Vorgehen erschien notwendig, da in reinen Lösungen entschieden höhere Fluoreszenzausbeuten gemessen werden. Außerdem wurden Reagenz- leerwerte, nicht oxidierte Leerwerte (Zugabe von Kaliumhexacyanoferrat[III] nach Reduktionsreagenz und "faded blanks" erstellt. Unter diesen Bedingungen waren alle drei Leerwerte für jede Methode gleich, aber auch die Unterschiede zwischen den Methoden waren gering, wenn die Messung unverzüglich vorgenommen wurde. Bei den Angaben in Tabelle 5 erfolgte die Nulleinstellung des Fluorometers mit einem Reagenzleerwert, die Leerwerte zeigten keine spezifische Fluoreszenz.

Die reacidifizierende Trihydroxyindolmethode (Tab. 5, Methode 2) erbringt gegenüber der alkalischen Trihydroxyindolmethode (Tab. 5, Methode 1) nur für Adrenalin eine Steigerung der Fluoreszenzausbeute. Die hier beschriebene Methode (Tab. 5, Methode 3) weist eine Verbesserung der Fluoreszenzausbeute auf etwa das 3-fache im Vergleich zu der alkalischen Trihydroxyindolmethode (Tab. 5, Methode 1) auf. Allgemein läßt sich sagen, daß die Fluoreszenzausbeuten in Eluaten geringer sind als in reinen Puffern und in Boratpuffer höhere Fluoreszenzausbeuten gefunden werden als in Acetat- oder Phosphatpuffer.

\section{$\alpha$-Methylnoradrenalin und $\alpha$-Methyldopa}

Da unter Therapie mit $\alpha$-Methyldopa Harnkatecholaminuntersuchungen mit den üblichen alkalischen Trihydroxyindolmethoden wegen falsch-hoher Werte für die Phäochromocytomausschlußdiagnostik nicht

Tab. 5. Fluoreszenzausbeute verschiedener TrihydroxyindolMethoden in arbiträren Fluoreszenzeinheiten

Jeder Probe wurden 55 pmol (10 ng) Adrenalin bzw. $59 \mathrm{pmol}(10 \mathrm{ng})$ Noradrenalin zugcgeben ( $\mathrm{n}$ je 6$)$. Die Methoden 1 und 3 wurden mit borsaurem Leereluat der Fertigsäulen durchgeführt, Methode 2 mit Leereluat nach (16). Probenansatz zu den Methoden:

1) $0,8 \mathrm{ml}$ borsaures Eluat, $0,2 \mathrm{ml}$ Phosphatpuffer 0,5 $\mathrm{mol} / \mathrm{l} \mathrm{pH} 6,5,0,05 \mathrm{ml} \mathrm{0,16} \mathrm{mmol} / 1$ Zinksulfat, $0,05 \mathrm{ml} \mathrm{7,59} \mathrm{mmol} / 1 \mathrm{Kaliumhexacyanoferrat} \mathrm{(III),}$ nach $3 \mathrm{~min} 0,2 \mathrm{ml} \mathrm{NaOH}(5 \mathrm{~mol} / 1)-113,4 \mathrm{mmol} / 1$ Ascorbinsäure $(9: 1, \mathrm{~V} / \mathrm{V})$. Gesamtvolumen $1,3 \mathrm{ml}$. Wellenlänge: Adrenalin $405 / 520 \mathrm{~nm}$, Noradrenalin $405 / 495 \mathrm{~nm}$.

2) $0,8 \mathrm{ml}$ essigsaures Eluat $1 \mathrm{~mol} / 1, \mathrm{pH} 2,85$ für Adrenalin, pH 5,8 für Noradrenalin, $0,05 \mathrm{ml}$ Kupferacetat $0,01 \mathrm{~mol} / 1$ bei Adrenalin, $0,05 \mathrm{ml}$ deionisiertes Wasser bei Noradrenalin, $0,05 \mathrm{ml} 7,59 \mathrm{mmol} / 1$ Kaliumhexacyanoferrat (III) nach $5 \mathrm{~min} 0,2 \mathrm{ml}$ Natronlauge $10 \mathrm{~mol} / 1-0,14 \mathrm{~mol} / 1$ Mercaptoäthanol in $1,59 \mathrm{~mol} / 1$ Natriumsulfit $(1: 1, \mathrm{~V} / \mathrm{V})$. Nach $4 \mathrm{~min} 0,2 \mathrm{ml}$ Essigsäure $10 \mathrm{~mol} / \mathrm{l}$. Gesamtvolumen $1,3 \mathrm{ml}$. Präzipitat bei Adrenalin kurz abzentrifugiert. Wellenlänge: Adrenalin 415/500 nm, Noradrenalin 400/485 nm.

3) Probenansatz für Methode 3 entspricht den Angaben diescr Arbeit.

\begin{tabular}{lcc}
\hline Methode & Adrenalin & Noradrenalin \\
\hline 1 & 40 & 45 \\
2 & 52 & 40 \\
3 & 127 & 170 \\
\hline
\end{tabular}


möglich sind, wurde der Einfluß dieses Pharmakons und seiner Metaboliten auf die reacidifizierende Trihydroxyindolmethode geprüft. Es wurde die Katecholaminexkretion bei 13 Patienten bestimmt, die mit 9,5 mmol ( 2 g) $\alpha$-Methyldopa täglich behandelt wurden. Wie in Tabelle 6 zu sehen ist, waren die Exkretionswerte für Adrenalin und Noradrenalin bei der reacidifizierenden Trihydroxyindolmethode im unteren Normbereich, bei der alkalischen Trihydroxyindolmethode (6) stark erhöht. Von den geprüften Metaboliten des $\alpha$-Methyldopa zeigte $\alpha$-Methylnoradrenalin eine spezifische Fluoreszenz von $6 \%$ des Noradrenalins (Tab. 6). Bei der Adrenalinbestimmung ( $\mathrm{pH} 2,85$, in Tab. 6 nicht aufgeführt) ergab $\alpha$-Methylnoradrenalin keine spezifische Fluoreszenz.

Tab. 6. Katecholaminbestimmung im Harn während $\alpha$-Methyldopa-Therapie $(2 \mathrm{~g} / \mathrm{d})$

Die Katecholaminbestimmung erfolgte aus denselben Eluaten $(n=13)$ mit der hier angegebenen reacidifizierenden Trihydroxyindolmethode und der alkalischen Trihydroxyindolmethode von Häggendal (6).

\begin{tabular}{lll}
\hline Methode & $: \quad \begin{array}{ll}\text { Adrenalin } \\
\text { nmol } / 24 \mathrm{~h} \\
(\overline{\mathrm{x}} \pm \mathrm{s})\end{array}$ & $\begin{array}{l}\text { Noradrenalin } \\
\text { nmol } / 24 \mathrm{~h} \\
(\overline{\mathrm{x}} \pm \mathrm{s})\end{array}$ \\
\hline
\end{tabular}

Reacidifizierende

Trihydroxyindolmethode

$27,3 \pm 19,1$

$98,1 \pm 62,1$

Alkalische

Trihydroxyindol-

methode

$1295,7 \pm 354,3$

$1428,4 \pm 405,4$

Um diese Befunde zu spezifizieren, wurde ein Poolurin mit $\alpha$-Methylnoradrenalin $(54,6 \mu \mathrm{mol} / \mathrm{l}, \mathrm{n}=4)$ aufgestockt. Während die Adrenalinbestimmung unbeeinflußt war, fand sich eine Vermehrung der Noradrenalinausbeute um 219,3 nmol/1. Das entspricht einer Wiederfindungsrate von nur $60 \%$ für $\alpha$-Methylnoradrenalin.

Bei einer mittleren täglichen $\alpha$-Methylnoradrenalinexkretion von $147 \mathrm{nmol}(18)$ ist deshalb mit einer fälschlichen Noradrenalin-Mehrbestimmung von $5,9 \mathrm{nmol} / \mathrm{Tag}$ zu rechnen.

\section{Spezifität}

Um die Spezifität der vorliegenden Methode zu ermitteln, wurden Adrenalin und Noradrenalin, deren Metabolite, chemisch verwandte Phenole und andere biogene Amine in Boratpuffer pH 7 geprüft (Tab. 7). Wenn die Fluoreszenzen von $1 \mathrm{nmol}$ Adrenalin und Noradrenalin auf je $100 \%$ gesetzt werden, findet man eine Fluoreszenz von $40 \%$ bei Metanephrin und Normetanephrin.

$\alpha$-Methylnoradrenalin hat noch eine Fluoreszenz von $6 \%$, die übrigen biogenen Amine und Phenole haben keine nennenswerte Fluoreszenz. Die Spezifität und die
Richtigkeit wird durch das Spektrum von Urineluaten und mit Katecholaminen aufgestockten Urineluaten nachgewiesen (Abb. 2).

\section{Rich tigkeit}

Vergleichende Untersuchungen von Adrenalin (0,55$1,64 \mu \mathrm{mol} / \mathrm{l})$ und Noradrenalin $(0,59-1,77 \mu \mathrm{mol} / 1)$ ohne und mit Ionenaustauschchromatographie ergaben folgende Wiederfindung (\%) durch die Säule (Mittelwert \pm Standardabweichung; $\overline{\mathrm{x}} \pm \mathrm{s})$ :

Adrenalin $74,1 \% \pm 4,3(n=12)$

Noradrenalin $75,2 \% \pm 3,6(n=12)$

\section{Präzision}

Zur Untersuchung wurde ein Poolurin (in der Serie) und ein Urin von einem Patienten mit einem Phäochromocytom (von Tag zu Tag) verwendet.

In der Serie $\quad(\bar{x} \pm s, n=20)$ :

Adrenalin $\quad 33,3 \pm 2,7 \mathrm{nmol} / 1 \quad \mathrm{VK} \%=8,1$

Noradrenalin $232,9 \pm 9,5 \mathrm{nmol} / 1 \quad$ VK $\%=4,1$

Von Tag zu Tag ( $\bar{x} \pm s, n=20)$ :

Adrenalin $\quad 681,2 \pm 30,6 \mathrm{nmol} / 1 \mathrm{VK} \%=4,5$

Noradrenalin $4933,1 \pm 124,7 \mathrm{nmol} / \mathrm{i}$ VK $\%=2,5$

Tab. 7. Fluoreszenzentwicklung von Adrenalin und Noradrenalin im Vergleich zu anderen Stoffen

Adrenalin wurde bei der Wellenlänge 415/490 nm (Anregung/Emission) auf 100 gesetzt, Noradrenalin wurde bei $390 / 470 \mathrm{~nm}$ auf 100 gesetzt. Je Probe wurden $1 \mathrm{nmol}$ Reinsubstanz in Boratpuffer 0,67 mol/1, pH 7,0, eingesetzt:

\begin{tabular}{|c|c|c|}
\hline \multirow[t]{2}{*}{ Substanz } & \multicolumn{2}{|c|}{$\begin{array}{l}\text { Wellenlänge } \\
\text { (Anregung/Emission } \\
(\mathrm{nm}) \text { ) }\end{array}$} \\
\hline & $415 / 488$ & $388 / 468$ \\
\hline Noradrenalin & 61,52 & 100,00 \\
\hline Adrenalin & 100,00 & 51,60 \\
\hline Normetanephrin & 25,05 & 39,12 \\
\hline Metanephrin & 37,70 & 12,57 \\
\hline$\alpha$-Methylnoradrenalin & 4,69 & 5,73 \\
\hline 3,4-Dihydroyphenylglycol & 0,33 & 0,14 \\
\hline 3-Methoxy-4-hydroxyphenyläthylamin & 0,26 & 0,62 \\
\hline$\alpha$-Methyl-3,4-dihydroxy-phenylalanin & 0,12 & 0,14 \\
\hline 3,4-Dihydroxyphenalalanin & 0,07 & 0,21 \\
\hline Serotonin & 0,03 & 0,04 \\
\hline Tyramin & 0,09 & 0,14 \\
\hline$L$-Histidin & 0,03 & 0,07 \\
\hline$D, L$-Tyrosin & 0,14 & 0,20 \\
\hline 3,4-Dihydroxyphenylessigsäure & 0,08 & 0,18 \\
\hline 3-Methoxy-4-hydroxyphenyläthylenglycol & 0,07 & 0,09 \\
\hline Histamin & 0,28 & 0,27 \\
\hline Vanillinsäure & 0,07 & 0,11 \\
\hline Vanillinmandelsäure & 0,32 & 0,40 \\
\hline Homovanillinsäure & 0 & 0 \\
\hline$D, L$-Cctopamin & 0,20 & 0,35 \\
\hline 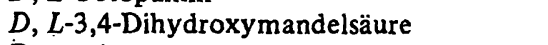 & 0,03 & 0,20 \\
\hline Dopamin & 0,25 & 0,48 \\
\hline
\end{tabular}



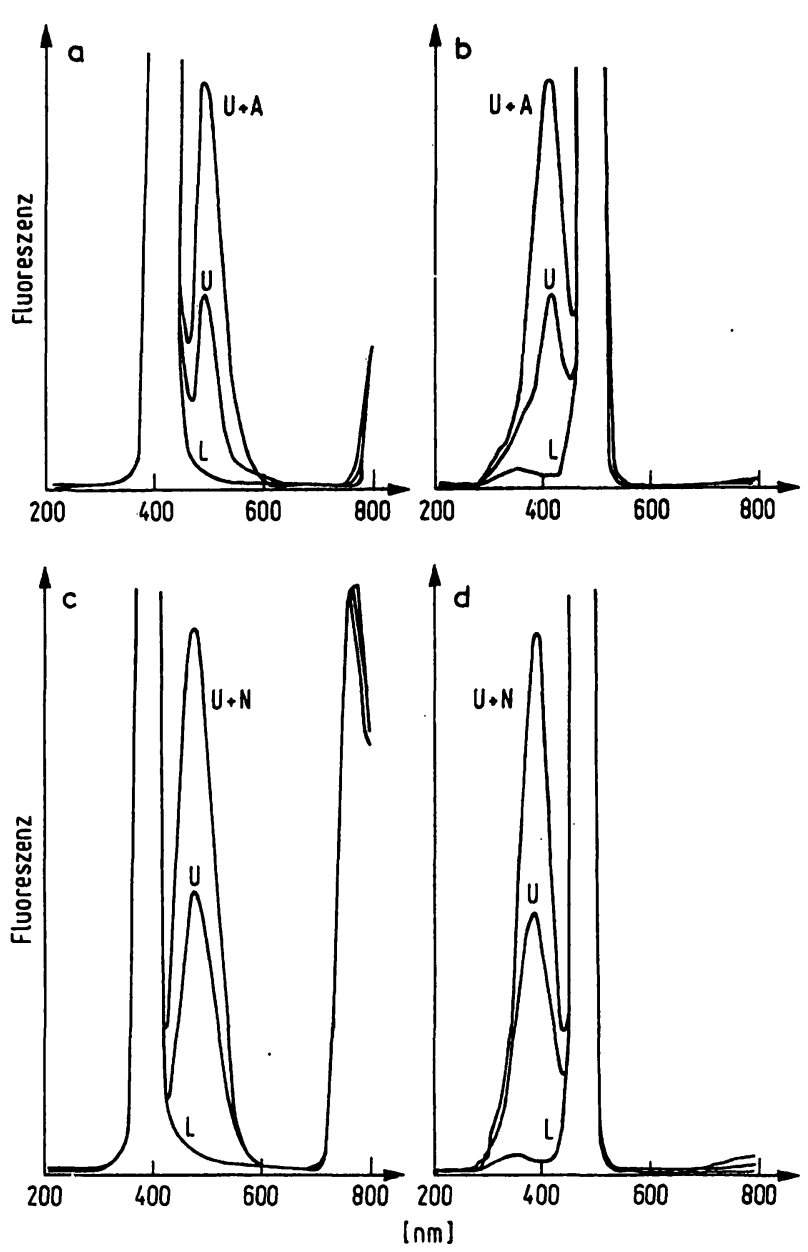

Abb. 2. Fluoreszenzspektrum. Urineluat (U), Leerwert (L), Adrenalin (A), Noradrenalin (N).

Die Eluate wurden ohne und mit aufgestocktem Amin (55 pmol Adrenalin oder $59 \mathrm{pmol}$ Noradrenalin) bestimmt. Wellenlängenangabe unkorrigiert.

a. Emissionsspektrum bei pH 2,85 und einer Anregung von $415 \mathrm{~nm}$.

b. Anregungsspektrum bci pH 2,85 und eincr Emission von $490 \mathrm{~nm}$.

c. Emissionsspektrum bei $\mathrm{pH} 7$ und einer Anregung von $390 \mathrm{~nm}$.

d. Anregungsspektrum bei pH 7 und einer Emission von $470 \mathrm{~nm}$.

Nach weisgrenze

Die Nachweisgrenze wurde gemäß Kaiser (10) aus dem Mittelwert des Leerwertes und der Standardabweichung errechnet $(\bar{x}+3 s)$.

Adrenalin $\quad 3,4 \mathrm{nmol} / 1$

Noradrenalin $16,3 \mathrm{nmol} / 1$

\section{Normalwerte}

Die Untersuchung umfaßt Harne von 17 normotonen, gesunden, im Arbeitsprozeß stehenden Probanden beiderlei Geschlechts.

Adrenalin $\quad(\bar{x} \pm s)=44,8 \pm 16,9 \mathrm{nmol} / 24 \mathrm{~h}$

Noradrenalin $(\bar{x} \pm s)=224,0 \pm 68,0 \mathrm{nmol} / 24 \mathrm{~h}$

Eine Normalverteilung ist bei Normotonikern zwar anzunehmen, konnte aber aufgrund der kleinen Probandenzahl nicht bewiesen werden.

\section{Diskussion}

Die chromatogiaphische Trennung erfährt eine wesentliche Erleichterung durch den Gebrauch von Fertigsäulen, auch gegenüber der Batch-Technik mit Aluminiumoxid. Bei den von uns bevorzugten Säulen wird Borsäure als Elutionsmittel gebraucht. Borsaures Eluat war die Voraussetzung für die hier beschriebene Trihydroxyindol-Bestimmungsmethode, bei der die Kombination von Borsäure, Kupferionen, Thiol und finaler Ansäuerung zu einer optimalen Fluoreszenzausbeute führt.

Zuerst berichteten Trautner \& Messer (11) über eine Schutzwirkung vor oxidativem Abbau der Katecholamine durch Borat. Gerst et al. (12) verwendeten Natriumborhydrid und erreichten damit eine Stabilisierung der Fluoreszenz in Probe und Leerwert. Mattok \& Wilson (13) und Valori et al. (14) berichten, daß Borsäure ein gutes Elutionsmittel für Katecholamine ist, wenn das schwache Kationenaustauschharz Amberlite CG 50 verwandt wird. $\mathrm{Zu}$ ähnlichen Ergebnissen kamen Sandhu \& Freed (15) beim Gebrauch von Bio-Rex 70.

Wenn Borsäure 0,67 mol/l die Fertigsäulen passiert, verändert sich der $\mathrm{pH}$ von 3,8 auf 7,0 . Noradrenalin hat jedoch ein Fluoreszenzmaximum, wenn es bei pH 5 oxidiert wird (Abb. 3). Da die Gefahren der Verschleppung, Verunreinigung, Verdünnung und der Zeitaufwand höher einzuschätzen sind als der Gewinn an Fluoreszenzausbeute von etwa $15 \%$, wurde bei den kleinen Volumina bewußt darauf verzichtet, den $\mathrm{pH}$ von 7,0 auf 5,0 zu bringen. Außerdem ist Boratpuffer bei pH 5 sehr labil und schwierig einzustellen. Obwohl die Eluate in der Regel sofort bestimmt wurden, können diese auch aufbewahrt werden. Werden die Eluate eingefroren, muß darauf geachtet werden, daß sie bis zur Bestimmung wieder Raumtemperatur angenommen haben, da sonst die Reaktion, besonders bei pH 2,85, nur unvollständig abläuft und Fehlbestimmungen die Folge sind. Auch sollte die fluorimetrische Bestimmung immer bei gleicher Temperatur erfolgen. Gerst et al. (12) haben nachweisen können, daß in Kälte die Fluoreszenz der Lutine zunimmt und in Wärme abnimmt. Dieser reversible Prozeß gilt auch für die vorliegende Methode und kann entsprechend verwertet werden. Allerdings beschlagen die kalten Küvetten häufig.

Weil-Malherbe \& Bigelow (7) steigerten die Fluoreszenzausbeute der Trihydroxyindolmethode, indem sie Kupferionen als Katalysator einsetzten. Ein vom Kupfer herrührendes Präzipitat, das nach der Zugabe von Essigsäure ausfällt, muß bei dieser Methode abzentrifugiert werden. Wisser (8) gab eine automatisierte Methode mit einem Auto-Analyzer an, die auf den Angaben von Weil-Malherbe \& Bigelow (7) beruhte. Er ließ die Zugabe von Essigsäure weg, um das Präzipitat nicht durch einen Dialyseschritt entfernen zu müssen. Allerdings ist 
die Fluoreszenzausbeute auch dementsprechend geringer (vgl. Tab. 3). Bei der vorliegenden Methode ist eine geringere Kupferkonzentration erforderlich und ein Präzipitat fällt nicht aus. Deshalb ist es möglich, diese Methode ohne Einbußen zu automatisieren (in Vorbereitung).

Wird Noradrenalin bei pH 7 oxidiert, führt der Zusatz von Kupferionen zu einer Steigerung der Fluoreszenzausbeute um $90 \%$ (Tab. 3). Diese Steigerung war in Acetatpuffer nicht möglich. Kupferionen haben zwei mögliche Funktionen: Erstens eine kataly tische Funktion während der Oxidation besonders für Adrenalin bei pH 2,85 und zweitens eine die Fluorophore stabilisierende Funktion (Abb. 1) (6). Die zweite Funktion weist auf eine mögliche Reaktion mit den Lutinen hin. Diese Reaktionen werden weiter kompliziert, da Borsäure die Fähigkeit zur Komplexbildung mit Katecholaminen hat (11).

So wie der Katecholamin-Borat-Komplex vor Luftsauerstoff geschützt ist, könnte auch ein TrihydroxyindolBorat-Komplex vor oxidativem Zerfall geschützt sein.

Die Differenzierung ist sicher optimal, wenn bei $\mathrm{pH}$ 2,85 in Gegenwart von Kupferionen ausschließlich Adrenalin, bei pH 7 ohne Kupfer fast ausschließlich Noradrenalin fluoreszierende Lutine bildet (Tab. 3). Diese Art der Differenzierung ist bei der vorliegenden Bestimmungsmethode möglich. Dennoch haben wie es in unserem Labor vorgezogen, auch bei pH 7 in Gegenwart von Kupferionen zu oxidieren. Die spezifische Fluoreszenz erhebt sich hierbei klar über die unspezifischen Begleitprodukte, die Fluoreszenzausbeute ist größer, die Fluoreszenzprodukte sind extrem stabil und eine genaue Bestimmung des kleinen Anteils, nämlich des Adrenalins, bleibt unberührt erhalten.

Noradrenalin wird bei pH 2,85 in der Regel nicht oxidiert. Weil-Malherbe (16) fand eine Oxidation von 0-3\%. Um gelegentlich bei der Adrenalinbestimmung aufgetretene Fehler zu eliminieren, bieten sich mehrere Möglichkeiten an. Erstens sollte die Molarität der Ameisensäure und der Oxidations-pH kontrolliert und die Bestimmung wiederholt werden. Dabei konnten in unserem Labor bei 5000 Untersuchungen alle Adrenalinbestimmungen exakt durchgeführt werden. $Z$ weitens kann die Oxidation bei pH 2,80 durchgefürht werden, indem eine konzentriertere Ameisensäure (2,1-2,4 $\mathrm{mol} / \mathrm{l})$ eingesetzt wird. Eine regelmäßige Kontrolle des Oxidations-pH ist ohnehin empfehlenswert, da bei den verschiedenen Chargen der Fertigsäulen geringe Verschiebungen des Eluat-pH vorkommen können. Drittens besteht die Möglichkeit, die Differenzierung der Amine nach einem der optischen Verfahren vorzunehmen. Die Trennungsgenauigkeit ist dann herabgesetzt. Die Differenzierungsgrenze eines Katecholamingemisches liegt etwa bei einem Verhältnis der Anteile Adrenalin zư Noradrenalin von $1: 5$ für das optische Verfahren und bei 1 : 20 für das 2-pH-Verfahren (9), wenn Kaliumhexacyanoferrat[III] als Oxidans verwendet wird. Die finale Reacidifizierung auf $\mathrm{pH} 4,5$ verbessert die Fluoreszenzausbeute, stabilisiert Haupt- und Leerwert und vermindert unspezifische Störeinflüsse 2. B. durch Serotonin und Histamin (17).

In unserem Labor ist es schon mehrfach vorgekommen, daß im Urin von Patienten mit einer Heppatopathie die Noradrenalinbestimmung mit einer alkalischen ThiolMethode (6) Werte um 800-1200 nmol/24 h ergab. Die simultan aus demselben Eluat durchgeführte Katecholaminbestimmung mit der vorliegenden Thiol-Methode ergab Normwerte. Wir fuihren diese gelegentlich auftretende Diskrepanz auf die Elimination unspezifischer Störeinflüsse zurück. Hier kamen diese möglicherweise aus dem Aminosäuremetabolismus. Eine andere Störung der Adrenalin- und Noradrenalinbestimmung tritt bei den gebräuchlichen Trihydroxyindolmethoden während der Behandlung mit $\alpha$-Methyldopa auf. Es kommt zur Exkretion beträchtlicher Mengen von $\alpha$-Methyldopa, $\alpha$-Methyldopamin und $\alpha$-Methylnoradrenalin (18) und es werden fälschlich hohe Katecholaminexkretionswerte gefunden, die an ein Phäochromocytom denken lassen.

Die vorliegende Methode wird durch $\alpha$-MethyldopaTherapie praktisch nicht gestört, die Exkretionswerte für Adrenalin und Noradrenalin sind erwartungsgemäß herabgesetzt (18).

Eine weitere Verfälschung des Bestimmungsergèbnisses scheint durch Metanephrin und Normetanephrin möglich (Tab. 6). Von der Säule werden nur die freien $\mathrm{O}$-methylierten Katecholamine zurückgehalten. Mit der Borsäure werden bei einem Elutionsvolumen bis $7,5 \mathrm{ml}$ keine, bis $10 \mathrm{ml} \mathrm{ca.} \%$ der freien Metanephrine eluiert. Da die täglichen Exkretionswerte nach Taniguchi et al. (19) für freies Metanephrin $147 \mathrm{nmol}$ und für freies Normetanephrin $114 \mathrm{nmol}$ betragen, ist mit einer Verfälschung des Adrenalin- und Noradrenalinwertes um etwa $1,7 \mathrm{nmol}$ zu rechnen. Normetanephrin bildet bei pH 2,85 kein Fluoreszenzprodukt. Die durch die freien Metanephrine bedingte Abweichung fällt somit noch in den Bereich der methodischen Streuung, wenn mit $10 \mathrm{ml}$ Borsäure eluiert wird. Die relativ komplizierte Katecholaminbestimmungsmethode wurde einer statistischen Qualitätskontrolle unterzogen. Die Ausbeute von $75 \%$ ist befrie digend. Die Präzision in der Serie, ausgedrückt als Variationskoeffizienz ( $\mathrm{V} \%$ ), kann bei einer Hormonbestimmungsmethode mit chromatographischer Trennung als gut angesehen werden, wenn sie unter $10 \%$ liegt.

Der șchwache Punkt einer Harnkatecholaminbestimmung ist die Adrenalinbestimmung. Hier war der Variationskoeffizient $8,1 \%$ bei der Bestimmung in der Serie. Der Adrenalingehalt je Probenanșatz betrug dabei nur 4,2 pmol. Würde statt $5 \mathrm{ml}$ Urin ein größeres 
Ausgangsvolumen gewählt, wäre der Variationskoeffizient noch zu verbessern. Ein solches Vorgehen ist wegen der begrenzten Bindungsfähigkeit des Harzes einerseits und dem hohen Salzgehalt des Urins andererseits nur bei Säulen mit einer größeren Harzeinwaage zu rechtfertigen. Die Bestimmung der Präzision von Tag zu Tag hatte einen niedrigen Variationskoeffizienten, da der Urin des Phäochromocy tompatienten einen hohen Katecholamingehalt hatte. Voraussetzung für die insgesamt günstigen Präzisionsangaben war die hohe Fluoreszenzausbeute der angewandten Methode.

\section{Literatur}

1. Udenfriend, S. (1964), Fluorescence Assay in Biology and Medicine, Academic Press, New York.

2. Lund, A. (1949), Acta Pharmacol. Toxicol. 5, 75-94.

3. von Euler, U. S. \& Floding, 1. (1955), Acta Physiol. Scand. 118, 45-56.

4. von Euler, U. S. \& Lishajko, F. (1961), Acta Physiol. Scand. 51, 348-356.

5. Palmer, J. F. (1964), West Indian Med. J. 13, 38-53.

6. Häggendal, J. (1963), Acta Physiol. Scand. 59, 242-254.

7. Weil-Malherbe, H. \& Bigelow, L. B. (1968), Anal. Biochem. $22,321-334$.

8. Wisser, H. (1970), Diese Z. 8, 637-648.

9. Crout, J. R. (1961), Standard Methods of Clinical Chemistry, Vol. 3, pp. 62-80. D. Seligson, Ed., Academic Press, New York.

10. Kaiser, H. (1965), Z. Anal. Chem. 209, 1-18.

11. Trautner, E. M. \& Messer, M. (1952), Nature (London) 169, $31-32$.
12. Gerst, E. C., Steinsland, O. S. \& Walscott, W. W. (1966), Clin. Chem. 12, 659-669.

13. Mattok, G. L. \& Wilson, D. L. (1965), Anal. Biochem. 11, 575-579.

14. Valori, C., Brunori, C. A., Renzini, V. \& Corea, L. (1970), Anal. Biochem. 33, 158-167.

15. Sandhu, R. S. \& Freed, R. M. (1968), Clin. Chem. 14, 824825.

16. Weil-Malherbe, H. (1968), Methods Biochem. Anal. 16, 302-315.

17. Price, H. L. \& Price, M. L. (1957), J. Lab. Clin. Med. 50, 769.

18. Muscholl, E. \& Rahn, K. H. (1968), Pharmacol. Clin. 1, 19-29.

19. Taniguchi, K., Kakimoto, Y. \& Armstrong, M. D. (1964), J. Lab. Clin. Med. 64, 469-484.

Dr. Udo Werner Medizinische Klinik und Poliklinik der Universität Essen (GHS) 43 Essen 1

Hufelandstraße 55 
